\title{
Physical activity, additional breast cancer events, and mortality among early-stage breast cancer survivors: findings from the WHEL Study
}

\author{
Lisa A. Cadmus Bertram • Marcia L. Stefanick - Nazmus Saquib • \\ Loki Natarajan - Ruth E. Patterson - Wayne Bardwell - Shirley W. Flatt • \\ Vicky A. Newman · Cheryl L. Rock · Cynthia A. Thomson · John P. Pierce
}

Received: 29 June 2010/ Accepted: 4 December 2010/Published online: 24 December 2010

(C) The Author(s) 2010. This article is published with open access at Springerlink.com

\begin{abstract}
Objective Research suggests that physical activity is associated with improved breast cancer survival, yet no studies have examined the association between post-diagnosis changes in physical activity and breast cancer outcomes. The aim of this study was to determine whether baseline activity and 1-year change in activity are associated with breast cancer events or mortality.

Methods A total of 2,361 post-treatment breast cancer survivors (Stage I-III) enrolled in a randomized controlled trial of dietary change completed physical activity measures at baseline and one year. Physical activity variables (total, moderate-vigorous, and adherence to guidelines) were calculated for each time point. Median follow-up was 7.1 years. Outcomes were invasive breast cancer events and all-cause mortality.

Results Those who were most active at baseline had a $53 \%$ lower mortality risk compared to the least active women $(\mathrm{HR}=0.47 ; 95 \%$ CI: $0.26,0.84 ; p=.01)$. Adherence to activity guidelines was associated with a
\end{abstract}

L. A. C. Bertram · N. Saquib · L. Natarajan ·

R. E. Patterson - W. Bardwell - S. W. Flatt .

V. A. Newman · C. L. Rock · J. P. Pierce $(\square)$

Moores UCSD Cancer Center, University of California,

3855 Health Sciences Drive, San Diego, La Jolla,

CA 94305-5411, USA

e-mail: jppierce@ucsd.edu

M. L. Stefanick

Stanford Prevention Research Center, Stanford University,

251 Campus Drive, Stanford, CA 94305-5411, USA

C. A. Thomson

Arizona Cancer Center, Department of Nutritional Sciences,

University of Arizona, 1177 E. 4th Street,

Tucson, AZ 85721, USA
$35 \%$ lower mortality risk $(\mathrm{HR}=0.65,95 \%$ CI: 0.47, 0.91 ; $p<.01)$. Neither baseline nor 1 -year change in activity was associated with additional breast cancer events.

Conclusions Higher baseline (post-treatment) physical activity was associated with improved survival. However, change in activity over the following year was not associated with outcomes. These data suggest that long-term physical activity levels are important for breast cancer prognosis.

Keywords Exercise - Recurrence - Survival - Behavior . Lifestyle

\section{Introduction}

Evidence suggests that physical activity may reduce the risk of developing breast cancer among post-menopausal women [1-5] and among the most physically active premenopausal women [6]. The Nurses' Health Study $(n=2,987)$ [7] and the Collaborative Women's Longevity Study $(n=4,482)$ [8] found that women who reported at least 3 metabolic equivalent task (MET) hours per week had significantly lower risk of death from all causes and from breast cancer. In two smaller cohorts of women recruited specifically because of prior early-stage breast cancer diagnosis, the Health, Eating, Activity, and Lifestyle (HEAL) study [9] and the Life After Cancer Epidemiology (LACE) study [10] (933 and 1,970 women, respectively), physical activity was shown to have a significant protective association with all-cause mortality. In these two studies, physical activity also tended to be associated with reduced risk of both recurrence and breast cancer death, which accounted for the majority of deaths. A recent report from the Norwegian Counties Study [5] 
reported a $64 \%$ decrease in all-cause mortality risk among post-menopausal breast cancer survivors in the highest versus lowest category of physical activity participation (recurrence was not examined). In a Canadian cohort of 1,233 women with incident breast cancer, risks of breast cancer death and death from all causes were also lower among the highest versus lowest quartiles of both moderate and vigorous intensity recreational activity. Additionally, pre-diagnosis recreational activity, particularly moderate intensity activity, had a beneficial association with survival after breast cancer [11].

Little is known about the effect of change in physical activity level on breast cancer prognosis. While the HEAL Study [9] analysis revealed a higher mortality risk of women who decreased their physical activity level from the year prior to diagnosis to 2 years post-diagnosis, no studies have examined the potential effect of post-diagnosis change in physical activity. Proposed mechanisms by which physical activity (and/or physical activity adoption) may improve breast cancer survival include reductions in circulating concentrations of estrogen, insulin and related growth factors, and inflammatory factors [12-14].

The Women's Healthy Eating and Living (WHEL) Study was a randomized controlled trial of the effect of a high-vegetable, fruit, and fiber diet on recurrence and overall survival conducted from 1995 to 2006 among 3,088 women who had completed treatment for Stage I ( $\geq 1 \mathrm{~cm})-$ IIIA breast cancer [15]. The low-fat, vegetable-rich WHEL dietary intervention did not produce significant differences in either breast cancer events $(16 \%)$ or deaths $(10 \%)$ from all causes compared to comparison conditions [16], except in a subset of women who were not experiencing hot flashes at baseline [17]. We previously reported that while physical activity alone did not significantly predict breast cancer events or all-cause mortality among the 1,490 WHEL participants assigned to the control group, there was a cluster effect for physical activity combined with fruit/vegetable intake. A 50\% reduction in mortality risk was observed among those who were highly physically active and ate five or more fruit/vegetable servings per day, regardless of adiposity [18]. In this report of the full WHEL cohort, we tested the hypothesis that higher levels of baseline physical activity alone and 1-year increases in physical activity would be associated with a reduction in additional breast cancer events and death from all causes.

\section{Methods}

Details of eligibility criteria, data collection, and breast cancer outcomes assessment and causes of death have been reported previously [16] and are briefly summarized here.
Participants

The WHEL trial participants were enrolled at 7 study sites between 1995 and 2000. Eligibility criteria included diagnosis of primary operable invasive stage I $(\geq 1 \mathrm{~cm})$, II, or III and breast carcinoma within the past 4 years; age 18-70 years at the time of diagnosis; no current or planned chemotherapy; no evidence of recurrent disease or new breast cancer since completion of initial treatment; and no other cancer in the past 10 years. Informed written consent was collected, and the Human Subjects Committee of all participating institutions approved the study procedures, and the data are publicly available.

Of the 3,088 WHEL participants, we required participants to complete physical activity assessments at both enrollment and at the 12-month time point. Accordingly, 727 of those in the WHEL Study were excluded from this analysis due to additional breast cancer events $<1$ year after randomization $(n=124)$, death $<1$ year after randomization $(n=2)$, loss to follow-up $(n=1)$, or incomplete physical activity data $(n=600)$. Of the remaining 2,361 women included in these analyses, there were 295 (12.5\%) breast cancer events and 163 (6.9\%) deaths from all causes over a median follow-up period of 7.1 years (range of 1.0 to 10.8 years) from enrollment into the trial.

\section{Data collection}

Physical activity was assessed by questionnaire at posttreatment baseline (at the time of enrollment into the trial) and at various follow-up points using a 9-item physical activity measure adapted from the Women's Health Initiative (WHI) [19]. The physical activity questionnaire assessed frequency, duration, and speed of walking outside the home and frequency and duration of participation in each of three intensity levels of exercise: mild, moderate, or strenuous (with example activities for each level). As previously presented [20], activity levels were converted into metabolic equivalent task (METs) min per week in accordance with Ainsworth's compendium of physical activities [21]: mild activity was assessed as 3 METs, moderate activity was assessed as 5 METs, and vigorous activity was assessed as 8 METs. For walking, slow, average, fast, and very fast were assessed as 2, 3, 4, and 6 METs, respectively. The physical activity measure was validated against an accelerometer and the widely accepted Physical Activity Recall (PAR) among a subset of 74 WHEL Study participants and was found to have validity and sensitivity comparable to that of the PAR [22]. Compared to the accelerometer, the WHI physical activity measure did not provide a significantly different estimate of physical activity (+6 min or $4 \%, p=.95)$.

At both the baseline and 1-year clinic visits, height and weight were measured using standard protocols and used to 
calculate body mass index $\left(\mathrm{BMI}, \mathrm{kg} / \mathrm{m}^{2}\right)$. Breast cancer characteristics (stage, number of positive lymph nodes, treatment) were verified using medical record review. Questionnaires or interviews were used to assess demographic characteristics (e.g., age, race/ethnicity, and education level), reproductive history (menopausal status, presence/absence of hot flashes), smoking status, and alcohol intake. The Rand SF-36 [23] was used to obtain measures of self-rated health and pain. Self-rated health was obtained from the single SF-36 item ("Would you say your health is..." with responses of "excellent," "very good," "fair," and "poor") then categorized as "poor/fair" vs. "good/very good/excellent." Pain was assessed using the SF-36's bodily pain subscale, which consists of two items-one assessing severity of pain and one assessing the degree to which pain interfered with daily work (either inside or outside the home). The reference period for both items was the past 4 weeks, and participants were asked to respond using a 5-point Likert scale. The two item scores were combined and adjusted for subscale score with a potential range of 0 (worst possible pain) to 100 (complete freedom from pain).

\section{Assessment of study outcomes}

Primary study end points for the WHEL Study were [1] breast cancer events, defined as the combined outcome of invasive breast cancer recurrence (local/regional or distal) or new primary breast cancer and [2] death due to any cause. Details regarding outcomes assessment have been published [16]. Briefly, during semi-annual telephone interviews, clinical staff queried participants about occurrence of outcome events, any hospitalization, or new or current medical diagnoses. Staff then conducted confirmation interviews for any report of a breast cancer event or death for which medical records and death certificates were obtained, and data were confirmed at study end for $>95 \%$ of participants. Follow-up time was censored at the time of the participant's death, at the last documented staff contact date, or at the study completion date (June 1, 2006).

The breast cancer event-free interval was defined as the time from date of enrollment (March, 1995-November, 2000) to development of an additional breast cancer event or study completion. The death-free interval was defined as the time from enrollment to reported/confirmed death or study completion.

\section{Statistical analysis}

\section{Physical activity}

Energy expenditure was calculated separately for mild activities (MET-level <3.0), moderate activities (METlevel 3.0-5.9), and vigorous activities (MET-level $\geq 6.0$ ).
A variable was also created for moderate-to-vigorous physical activity (MET-level $\geq 3.0$ ), which was then used to create a dichotomous variable ("meeting PA guideline") based on a cutoff of 10.0 MET-h/week, which equates to $150 \mathrm{~min} /$ week of moderate-pace walking or the equivalent amount of other exercise durations/intensities. This cutpoint is consistent with American College for Sports Medicine's (ACSM) current aerobic activity recommendation for adults aged $\geq 65$ or for those aged 50-64 years who have chronic conditions [24]. The following variables were created to describe the change in physical activity from baseline to 1 year: change in adherence to guidelines, change in total physical activity (MET-h/week), and change in moderate-to-vigorous physical activity (MET-h/ week). Univariate analyses were used to define quintiles of baseline physical activity (both total and moderate-vigorous) and change in total and moderate-vigorous physical activity from baseline to 1 year.

\section{Covariates}

Potential covariates included age categories $(<44,45-54$, $55-65$, and $>65$ years), race/ethnicity (non-Hispanic white, African-American, Hispanic, Asian-American, and other), education (college graduate: yes, no), fruit/vegetable consumption $(<5$ servings per day or $\geq 5$ servings per day, excluding potatoes and iceberg lettuce), menopausal status (pre-menopausal, peri-menopausal, and post-menopausal), smoking status (current, past, and never smokers), alcohol intake [none, 1-19 g/day (equivalent to $<2$ drinks/day), and $\geq 20 \mathrm{~g} /$ day], hot flashes at baseline (yes, no), and obesity status as normal weight $\left(<25 \mathrm{~kg} / \mathrm{m}^{2}\right)$, overweight $\left(25-29.9 \mathrm{~kg} / \mathrm{m}^{2}\right)$, or obese $\left(\geq 30 \mathrm{~kg} / \mathrm{m}^{2}\right)$. Cancer treatment and tumor characteristics included chemotherapy use (yes, no), tamoxifen or other anti-estrogen use (yes, no), tumor type (either or both lobular and ductal invasive, none), tumor differentiation (well, moderate, poor), and cancer stage. Given that recruitment occurred from 1995 to 2000, use of aromatase inhibitors was negligible among WHEL Study participants and is therefore not included as a covariate.

\section{Analyses}

Cox models were used to determine both unadjusted and adjusted associations between each of the predictor variables and each of the two outcomes (additional breast cancer events, all-cause mortality), and chi-square tests were conducted to examine the relationship between physical factors (BMI, cancer characteristics, self-rated health, bodily pain) and adherence to physical activity guidelines. Kaplan-Meier plots were use to plot all-cause mortality stratified by (a) baseline physical activity and, 
in a separate plot, (b) change in physical activity from baseline to 1 year. All tests were two-sided and analyses were conducted in SAS version 9.1 (Cary, NC).

\section{Results}

The cohort for these analyses consisted of 2,361 WHEL Study participants who were alive and recurrence-free at 1-year follow-up and had completed the 1-year physical activity questionnaire. Of these, 295 later experienced an additional breast cancer event and 195 died of any cause.

Baseline characteristics of the total cohort of 2,361 women, stratified by baseline adherence to physical activity guidelines, are shown in Table 1. Approximately one-half of the participants were under 55 years of age, college graduates, and overweight or obese (BMI $\left.\geq 25.0 \mathrm{~kg} / \mathrm{m}^{2}\right)$. Almost $90 \%$ were non-Hispanic white and $81 \%$ were postmenopausal at baseline.

Baseline physical activity level ranged widely with the average participant reporting $14.9(\mathrm{SD}=14.7)$ MET-h/ week of physical activity. Moderate-vigorous activity accounted for $80 \%$ of total activity. Approximately onehalf of participants were meeting physical activity guidelines at baseline and at 1-year follow-up. Participants who met physical activity guidelines at baseline were slightly older, leaner, less likely to be post-menopausal, less likely to smoke cigarettes or to have formerly smoked, and more likely to be non-Hispanic white and to have a college degree $(p<.05$ for all) than those who did not meet the guidelines. They also had a lower tumor stage at the time of breast cancer diagnosis, were less likely to have received chemotherapy, had fewer positive lymph nodes, and were more likely to use anti-estrogens $(p=.05$ for all). Of the 1,175 participants who were meeting the physical activity guideline at baseline, the vast majority ( $n=962 ; 82 \%$ ) were still meeting the guideline at 1 year. Of the 1,186 women who were not meeting the guideline at baseline, $899(76 \%)$ were still not meeting the guideline at 1 year.

Physical activity and additional breast cancer events

Neither baseline physical activity nor baseline-to-1-year change in physical activity was significantly associated with additional breast cancer events. It was observed that those in the highest quintile of moderate-vigorous baseline physical activity had a $33 \%$ fewer additional events compared to those in the lowest quintile $(p<.05)$. Given that the trend across quintiles was not significant, this may be the result of type I error. Alternatively, these observations may indicate a threshold effect, so that physical activity has a protective effect on additional breast cancer events only when the activity is performed at sufficient intensity and in relatively high amounts.

Of the 1,186 women who were not meeting physical activity guidelines at baseline, only a very small number $(n=213 ; 4.1 \%)$ increased physical activity and met guidelines at 1 year. Unexpectedly, this group was associated with higher risk of additional breast cancer events $(p=.04)$, although as such an increased risk was not observed when the outcome was mortality, this finding might be an artifact.

Physical activity and all-cause mortality

Baseline physical activity was associated with reduced mortality risk (Table 2). Women who met physical activity guidelines for aerobic activity at baseline experienced a $35 \%$ reduction in risk of mortality relative to those who did not meet the guideline (see Fig. 1a for Kaplan-Meier curve). Similarly, those in the highest quintile of total physical activity ( $\geq 24.7$ MET-h/week, or the equivalent of at least $60 \mathrm{~min}$ of brisk walking, 5 days per week) experienced a $53 \%$ mortality risk reduction relative to those in the lowest quintile. The association was even more pronounced when physical activity was limited to that of moderate or vigorous intensity, with those in the most active quintile at $61 \%$ lower risk than those in the least active category.

Only one significant association was observed for baseline-to-1-year change in physical activity and all-cause mortality. Those women who were meeting physical activity guidelines both at baseline and at 1-year follow-up experienced a $40 \%$ reduction in mortality risk (see Fig. $1 \mathrm{~b}$ for Kaplan-Meier curve). Those who were meeting physical activity guidelines at only one time point fared no better than those who were meeting the guidelines at neither baseline nor 1-year follow-up.

\section{Sensitivity analyses}

The observed association between higher baseline physical activity and lower all-cause mortality could be an artifact caused by low-baseline physical activity among women with latent disease. To address that issue, we re-ran our adjusted models to the data after excluding 62 women for whom deaths, additional breast cancer events, or censoring occurred within 24 months of randomization. This resulted in a reduced cohort of 2,299 participants, including 122 allcause deaths. Results were highly comparable to those in Table 2 and are therefore not presented.

Based on the previous published finding that, among WHEL Study control group participants [18], physical activity was only associated with improved outcomes 
Table 1 Baseline characteristics of WHEL participants $(N=2,361)$ stratified by adherence to physical activity guidelines

\begin{tabular}{|c|c|c|c|c|c|}
\hline & \multicolumn{2}{|c|}{ Meeting guidelines } & \multicolumn{2}{|c|}{ Not meeting guidelines } & \multirow[t]{2}{*}{$p$ for difference } \\
\hline & $N$ & Mean (SD) or $\%$ & $N$ & Mean (SD) or \% & \\
\hline Age, mean (SD) & 1,175 & $54.3(9.1)$ & 1,186 & $53.5(8.6)$ & .04 \\
\hline \multicolumn{6}{|l|}{ Age group (\%) } \\
\hline$<44$ & 154 & $13.1 \%$ & 155 & $13.1 \%$ & \multirow[t]{4}{*}{.02} \\
\hline $45-54$ & 434 & $36.9 \%$ & 499 & $42.1 \%$ & \\
\hline $55-65$ & 411 & $35.0 \%$ & 395 & $33.3 \%$ & \\
\hline$>65$ & 176 & $15.0 \%$ & 137 & $11.6 \%$ & \\
\hline \multicolumn{6}{|l|}{ Race/ethnicity } \\
\hline Non-Hispanic White & 1,055 & $89.8 \%$ & 988 & $83.3 \%$ & \multirow[t]{5}{*}{$<.0001$} \\
\hline African-American & 23 & $2.0 \%$ & 47 & $4.0 \%$ & \\
\hline Hispanic-American & 40 & $3.4 \%$ & 70 & $5.9 \%$ & \\
\hline Asian-American & 37 & $3.2 \%$ & 45 & $3.8 \%$ & \\
\hline Other & 20 & $1.7 \%$ & 36 & $3.0 \%$ & \\
\hline College graduate $(\%)$ & 746 & $63.5 \%$ & 562 & $47.4 \%$ & $<.0001$ \\
\hline BMI, mean $\mathrm{kg} / \mathrm{m}^{2}(\mathrm{SD})$ & 1,175 & $25.6(4.7)$ & 1,186 & $28.5(6.6)$ & $<.0001$ \\
\hline \multicolumn{6}{|l|}{ BMI category $(\%)$} \\
\hline$<25.0$ & 617 & $52.5 \%$ & 422 & $35.6 \%$ & \multirow[t]{3}{*}{$<.0001$} \\
\hline $25-29.9$ & 375 & $31.9 \%$ & 361 & $30.4 \%$ & \\
\hline$\geq 30.0$ & 183 & $15.6 \%$ & 403 & $34.0 \%$ & \\
\hline \multicolumn{6}{|l|}{ Menopausal status } \\
\hline Post-menopausal & 934 & $79.5 \%$ & 970 & $81.8 \%$ & \multirow[t]{3}{*}{$<.0001$} \\
\hline Pre-menopausal & 125 & $10.6 \%$ & 107 & $9.0 \%$ & \\
\hline Peri-menopausal & 114 & $9.7 \%$ & 109 & $9.2 \%$ & \\
\hline Hot flashes $(\%)$ & 349 & $29.7 \%$ & 357 & $30.1 \%$ & .98 \\
\hline \multicolumn{6}{|l|}{ Time since diagnosis } \\
\hline$<2$ years & 638 & $54.3 \%$ & 652 & $55.0 \%$ & \multirow[t]{2}{*}{.74} \\
\hline $2-4$ years & 537 & $45.7 \%$ & 534 & $45.0 \%$ & \\
\hline \multicolumn{6}{|l|}{ Tumor stage $(\%)$} \\
\hline I & 514 & $43.7 \%$ & 443 & $37.4 \%$ & \multirow[t]{5}{*}{.003} \\
\hline IIA & 378 & $32.2 \%$ & 396 & $33.4 \%$ & \\
\hline IIB & 121 & $10.3 \%$ & 172 & $14.5 \%$ & \\
\hline IIIA & 131 & $11.2 \%$ & 135 & $11.4 \%$ & \\
\hline IIIC & 31 & $2.6 \%$ & 40 & $3.4 \%$ & \\
\hline Chemotherapy (\%) & 759 & $64.6 \%$ & 852 & $71.8 \%$ & .0008 \\
\hline Radiation (\%) & 720 & $61.3 \%$ & 734 & $61.9 \%$ & .95 \\
\hline \multicolumn{6}{|l|}{ \# positive nodes (\%) } \\
\hline 0 & 730 & $62.1 \%$ & 671 & $56.6 \%$ & \multirow[t]{3}{*}{.03} \\
\hline $1-3$ & 304 & $25.9 \%$ & 365 & $30.8 \%$ & \\
\hline$>3$ & 141 & $12.9 \%$ & 149 & $12.6 \%$ & \\
\hline Anti-estrogen use (\%) & 836 & $71.5 \%$ & 759 & $65.0 \%$ & .0007 \\
\hline Smoking status & 636 & $54.1 \%$ & 663 & $55.9 \%$ & \multirow[t]{4}{*}{.01} \\
\hline Never & 508 & $43.2 \%$ & 468 & $39.5 \%$ & \\
\hline Former & 31 & $2.6 \%$ & 55 & $4.6 \%$ & \\
\hline \multicolumn{5}{|l|}{ Current } & \\
\hline \multicolumn{6}{|l|}{ Alcohol (\%) } \\
\hline 0 & 339 & $28.9 \%$ & 380 & $32.1 \%$ & \multirow[t]{3}{*}{.21} \\
\hline $1-19 \mathrm{~g} /$ day & 786 & $67.0 \%$ & 761 & $64.3 \%$ & \\
\hline $20+$ g/day & 48 & $4.1 \%$ & 42 & $3.6 \%$ & \\
\hline
\end{tabular}


Table 2 Adjusted analyses for physical activity, change in activity level additional breast cancer events, and death from all causes in a cohort of women with a history of breast cancer $(n=2,361)$

\begin{tabular}{|c|c|c|c|c|c|c|c|c|c|}
\hline \multirow[t]{2}{*}{ Physical activity (MET-h/week) } & \multirow[t]{2}{*}{$N$} & \multirow[t]{2}{*}{$n$} & \multicolumn{3}{|c|}{ Additional breast cancer events } & \multirow[t]{2}{*}{$n$} & \multicolumn{2}{|c|}{ All-cause mortality } & \multirow[t]{2}{*}{$p$ (trend) } \\
\hline & & & HR $(95 \% \mathrm{CI})$ & $p$ & $p$ (trend) & & HR $(95 \% \mathrm{CI})$ & $p$ & \\
\hline \multicolumn{10}{|l|}{ Baseline activity level } \\
\hline Total PA (continuous) & 2,361 & 295 & $0.99(0.99,1.00)$ & .21 & & 163 & $0.98(0.96,0.99)$ & .003 & \\
\hline \multicolumn{10}{|l|}{ Total PA (categorical) } \\
\hline Quintile 1 [0-2.5] & 481 & 69 & Reference & & .58 & 43 & Reference & & .08 \\
\hline Quintile 2 (2.5-7.5] & 485 & 65 & $0.91(0.64,1.28)$ & .58 & & 43 & $1.01(0.66,1.55)$ & .97 & \\
\hline Quintile 3 (7.5-14.9] & 451 & 53 & $0.85(0.59,1.22)$ & .38 & & 32 & $0.85(0.53,1.35)$ & .48 & \\
\hline Quintile 4 (14.9-24.7] & 472 & 60 & $0.97(0.68,1.39)$ & .87 & & 27 & $0.75(0.46,1.23)$ & .26 & \\
\hline Quintile 5 (24.7-107] & 472 & 48 & $0.74(0.50,1.10)$ & .13 & & 18 & $0.47(0.26,0.84)$ & .01 & \\
\hline Mod-vig PA (continuous) & 2,361 & 295 & $0.99(0.98,1.00)$ & .13 & & 163 & $0.98(0.96,0.99)$ & .002 & \\
\hline \multicolumn{10}{|l|}{ Mod-vig PA (categorical) } \\
\hline Quintile 1 [0-1.3] & 474 & 70 & Reference & & .30 & 45 & Reference & & .02 \\
\hline Quintile 2 (1.3-6.3] & 473 & 64 & $0.97(0.68,1.36)$ & .84 & & 38 & $1.00(0.64,1.55)$ & .99 & \\
\hline Quintile 3 (6.3-12.5] & 474 & 64 & $0.98(0.69,1.38)$ & .89 & & 41 & $0.99(0.64,1.54)$ & .96 & \\
\hline Quintile 4 (12.5-22.9] & 470 & 53 & $0.91(0.62,1.31)$ & .60 & & 24 & $0.71(0.42,1.19)$ & .19 & \\
\hline Quintile 5 (22.9-107] & 470 & 44 & $0.67(0.45,1.00)$ & .04 & & 15 & $0.39(0.21,0.72)$ & .003 & \\
\hline \multicolumn{10}{|l|}{ Meeting PA guideline $^{\mathrm{a}}$} \\
\hline No & 1,186 & 161 & Reference & & & 102 & Reference & & \\
\hline Yes & 1,175 & 134 & $0.89(0.70,1.14)$ & .36 & & 61 & $0.65(0.47,0.91)$ & .01 & \\
\hline \multicolumn{10}{|l|}{ Baseline-to-1-year change } \\
\hline \multicolumn{10}{|l|}{ Meeting PA guideline } \\
\hline $\mathrm{No} \rightarrow \mathrm{No}$ & 899 & 112 & Reference & & & 75 & Reference & & \\
\hline No $\rightarrow$ Yes & 287 & 49 & $1.44(1.02,2.03)$ & .04 & & 27 & $1.21(0.77,1.90)$ & .42 & \\
\hline Yes $\rightarrow$ No & 213 & 31 & $1.22(0.81,1.83)$ & .34 & & 17 & $1.04(0.61,1.77)$ & .89 & \\
\hline Yes $\rightarrow$ Yes & 962 & 103 & $0.93(0.70,1.24)$ & .62 & & 44 & $0.60(0.40,0.88)$ & .01 & \\
\hline$\Delta$ Total PA (continuous) & 2,361 & 295 & $1.01(1.00,1.02)$ & .26 & & 163 & $1.00(0.98,1.01)$ & .59 & \\
\hline \multicolumn{10}{|l|}{$\Delta$ Total PA (categorical) } \\
\hline Quintile $1[-68.8,-5.5]$ & 474 & 52 & Reference & & .60 & 23 & Reference & & .70 \\
\hline Quintile $2(-5.5,-0.3]$ & 473 & 58 & $0.95(0.64,1.42)$ & .81 & & 39 & $1.24(0.71,2.16)$ & .45 & \\
\hline Quintile $3(-0.3,2.3]$ & 474 & 65 & $1.24(0.82,1.87)$ & .31 & & 40 & $1.23(0.69,2.20)$ & .49 & \\
\hline Quintile $4(2.3,7.5]$ & 470 & 57 & $1.10(0.73,1.65)$ & .66 & & 35 & $1.17(0.66,2.09)$ & .60 & \\
\hline Quintile $5(7.5,92.3]$ & 470 & 63 & $1.19(0.80,1.77)$ & .39 & & 26 & $0.89(0.49,1.64)$ & .71 & \\
\hline$\Delta$ Mod-vig PA (continuous) & 2,361 & 295 & $1.01(1.00,1.02)$ & .23 & & 163 & $0.99(0.98,1.01)$ & .48 & \\
\hline \multicolumn{10}{|l|}{$\Delta$ Mod-vig PA (categorical) ${ }^{b}$} \\
\hline Quintile $1[-63.8,-5.3]$ & 476 & 54 & Reference & & .58 & 24 & Reference & & .84 \\
\hline Quintile $2(-5.3,0]$ & 805 & 99 & $0.95(0.65,1.40)$ & .76 & & 69 & $1.17(1.69,1.97)$ & .56 & \\
\hline Quintile $3(0,1.3]$ & 147 & 18 & $1.00(0.57,1.75)$ & .99 & & 9 & $0.90(0.40,2.02)$ & .81 & \\
\hline Quintile $4(1.3,7)$ & 563 & 55 & $0.99(0.66,1.49)$ & .97 & & 32 & $1.06(0.60,1.89)$ & .84 & \\
\hline Quintile $5[7,92.3]$ & 470 & 69 & $1.23(0.83,1.80)$ & .30 & & 29 & $0.92(0.51,1.66)$ & .78 & \\
\hline
\end{tabular}

All analyses adjusted for age at randomization, race, fruit and vegetable consumption, BMI at randomization, menopausal status, tumor type, tumor grade, tumor stage, anti-estrogen use, clinical site, time from diagnosis to randomization, hot flashes, and study group

a To approximate guideline of $150 \mathrm{~min} / \mathrm{wk}$, we used a cutoff of $10 \mathrm{MET}-\mathrm{h} /$ week of moderate-to-vigorous PA

${ }^{b}$ Quintiles are uneven due to a large number of participants with change scores of exactly 0

among women who also reported high fruit/vegetable intake ( $\geq 5$ servings/day), we tested the full sample (control and intervention women) for such a relationship. No interaction was observed; therefore, the presented findings include fruit/vegetable consumption as a first-order covariate, but not as part of an interaction term. 


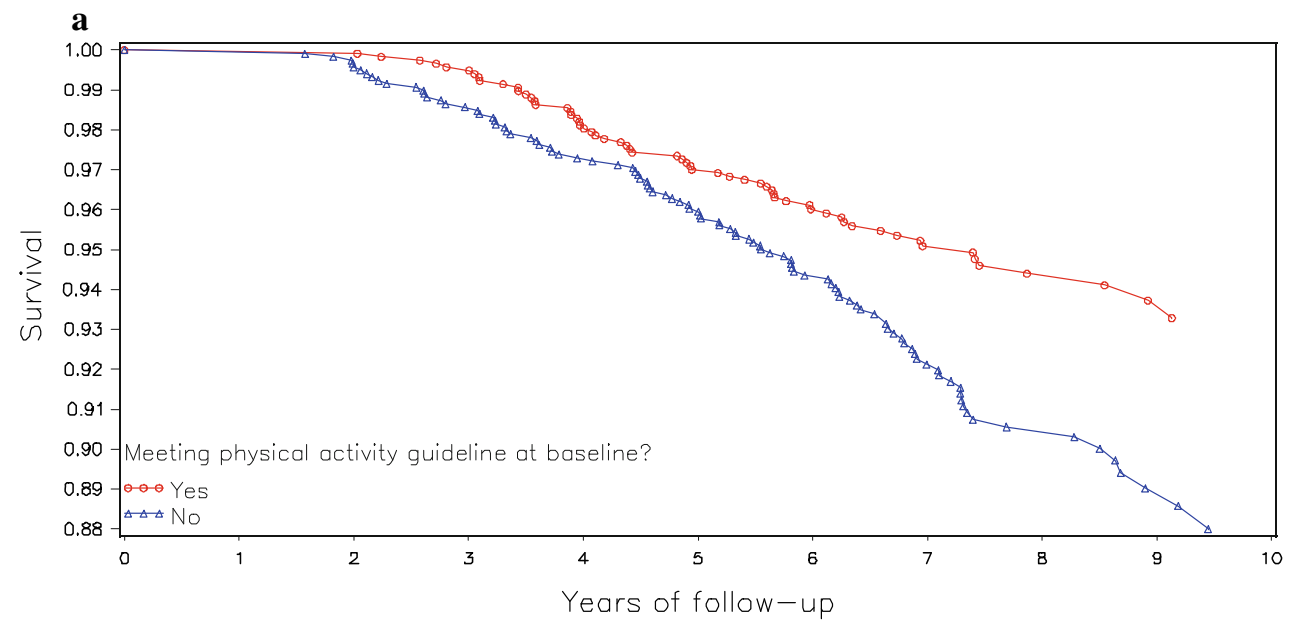

b

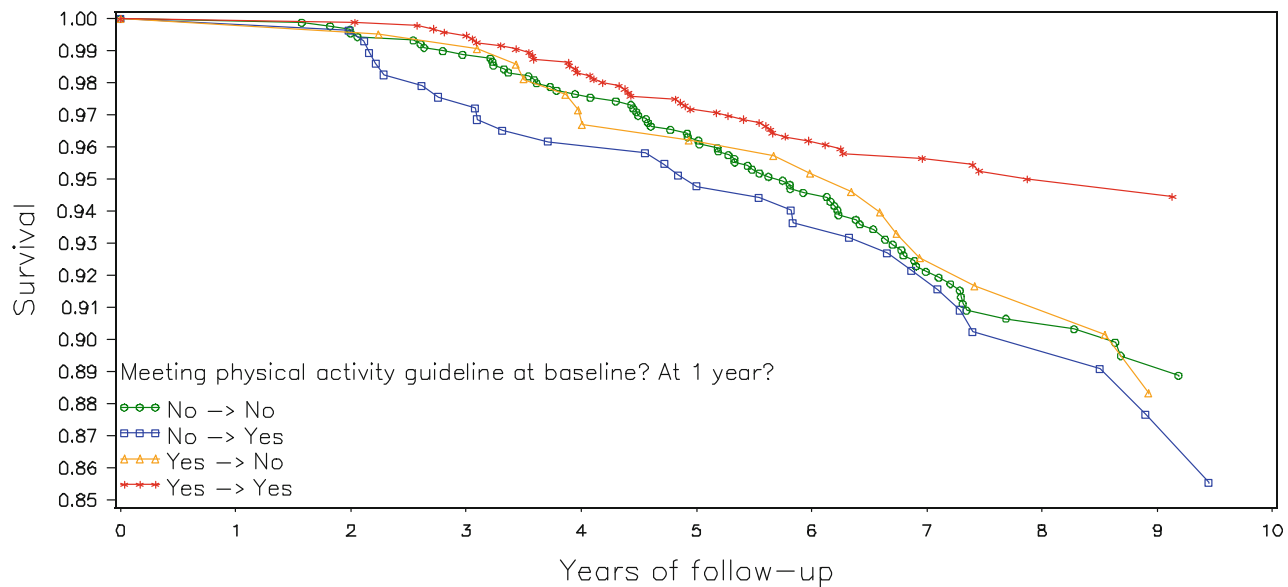

Fig. 1 a All-cause mortality for WHEL Study participants $(N=2,361)$ stratified by baseline physical activity level. b All-cause mortality stratified by change in physical activity from baseline to 1 year

WHEL Study participants were recruited up to 4 years after diagnosis. To address the possibility that it was the physical activity level shortly after diagnosis that mattered, we conducted a sensitivity analysis using only the 1,071 women who were diagnosed $<2$ years prior to randomization. The only result to change was that meeting physical activity guidelines at baseline was now associated with reduced breast cancer events (HR $=0.71 ; 95 \% \mathrm{CI}$ : 0.51 , $0.98 ; p=.04)$.

Similarly, removing low-risk women (i.e., Stage I) from the analysis reduced the sample size to 1,404 breast cancer survivors. This subgroup analysis did not alter effect sizes, however, it reduced study power to identify those effect sizes as significant.

\section{Discussion}

We found a robust association between level of physical activity at baseline and breast cancer mortality. We did not observe a statistically significant association between baseline physical activity and additional breast cancer events, suggesting that the results for mortality may have been driven by prognosis after an additional event (including a possible effect on type of recurrence) rather than a direct reduction in recurrences or new primaries. Although our analyses used all-cause rather than breast cancer-specific mortality, more than $80 \%$ of deaths in the WHEL Study were due to breast cancer [16]; therefore, the observed association between higher baseline physical activity and lower mortality was largely driven by a reduction in breast cancer-related deaths.

Contrary to expectations, changes in physical activity from baseline to 1 year were not associated with survival. It is possible that our 1-year measure of behavior change did not provide a sufficiently long period for the metabolic and physiologic changes associated with increased or decreased physical activity to impact cancer outcomes. Likewise, we observed that the majority of women who adopted physical activity between enrollment and 
year 1 did not maintain their new activity level over the long term.

Our findings with regard to mortality are generally consistent with previous reports [7, 8]. Similar to the Nurses' Health Study, the Collaborative Women's Longevity Study, and the Life after Cancer Epidemiology cohorts, we found that a substantial amount of physical activity was required to improve breast cancer outcomes. An exception is the HEAL Study, which observed a benefit for even very modest levels of physical activity. There are a number of potential explanations for between-study differences in the level of physical activity that is associated with reduced mortality. The cohorts vary somewhat with regard to clinical and other participant characteristics. Perhaps more importantly, the specific approach to physical activity measurement and quantification also varies. For example, the Canadian study assessed a wide variety of physical activity settings (recreational, household, occupational) and categorized results by setting type, whereas some other studies focused only on recreational or leisuretime activity and categorized results by intensity (mild, moderate, vigorous, total). Due to these variations, it is difficult to directly compare studies with regard to the dose of physical activity that is necessary to observe a protective effect on outcomes.

A strength of the WHEL Study was that we assessed physical activity at two time points, allowing us to assess the potential effect of changes in physical activity on breast cancer outcomes. Other strengths included excellent cohort maintenance (health status confirmed at study end confirmed for $>95 \%$ of participants) and a multi-site, geographically diverse sample of breast cancer survivors.

A limitation of the WHEL Study was the use of selfreport of physical activity, which is known to include random error. However, the study used a standardized physical activity questionnaire that was validated against both a physical activity recall procedure and an objective physical activity measure (7-day accelerometer) within a subset of WHEL Study participants [22]. The physical activity questionnaire had good agreement (73\%) with the accelerometer measure and had $100 \%$ sensitivity for meeting the physical activity guideline. Another limitation is that the WHEL cohort was a fairly racially and ethnically homogenous sample. Thus, findings may not apply to women who are not non-Hispanic White.

Consistent with previous reports, our findings suggest that adherence to physical activity guidelines may improve overall survival among breast cancer survivors. We also present the first examination of post-diagnosis change in physical activity. We observed a $42 \%$ reduction in risk mortality among those women who were adhering to physical activity guidelines at both baseline and 1-year relative to those who did not meet the guideline at either time point.
However, we did not find that 1-year change in physical activity was associated with breast cancer mortality. These findings highlight the importance of sustained post-diagnosis physical activity and suggest that a 1-year period may be too short for even relatively large increases in physical activity to affect breast cancer outcomes. Long-term exposure appears to be the most important determinant of the relationship between physical activity and breast cancer mortality. To improve breast cancer outcomes, women should be encouraged to maintain an active lifestyle over time, aiming to meet or exceed current physical activity guidelines. Toward this goal, physical activity promotion should be incorporated into breast cancer prevention and control programs. Future studies are needed to further examine the potential effect of long-term post-diagnosis change in physical activity on breast cancer outcomes.

Acknowledgments The Women's Healthy Eating and Living (WHEL) Study was initiated with the support of the Walton Family Foundation and continued with funding from National Cancer Institute Grant CA 69375. Some of the data were collected from General Clinical Research Centers, National Institute of Health grants M01RR00070, M01-RR00079, and M01-RR00827.

Open Access This article is distributed under the terms of the Creative Commons Attribution Noncommercial License which permits any noncommercial use, distribution, and reproduction in any medium, provided the original author(s) and source are credited.

\section{References}

1. Thune I, Furberg AS (2001) Physical activity and cancer risk: dose-response and cancer, all sites and site-specific. Med Sci Sports Exerc 33(6 Suppl):S530-S550 discussion S609-10

2. Sternfeld B, Lee I-M (2008) Physical activity and cancer: the evidence, the issues, and the challenges. In: Lee I-M (ed) Epidemiologic methods in physical activity studies. Oxford University Press, New York

3. Bull FC, Armstrong TP, Dixon T, Ham S, Neiman A, Pratt M (2004) Physical inactivity. In: Ezzati M, Lopez AM, Rodgers A, Murray C (eds) Comparative quantification of health risks: global and regional burden of disease attribution to selected major risk factors. World Health Organization, Geneva

4. Monninkhof EM, Elias SG, Vlems FA et al (2007) Physical activity and breast cancer: a systematic review. Epidemiology 18(1):137-157

5. Emaus A, Veierod MB, Tretli S et al (2010) Metabolic profile, physical activity, and mortality in breast cancer patients. Breast Cancer Res Treat 121(3):651-660

6. John EM, Horn-Ross PL, Koo J (2003) Lifetime physical activity and breast cancer risk in a multiethnic population: the San Francisco Bay area breast cancer study. Cancer Epidemiol Biomarkers Prev 12(11 Pt 1):1143-1152

7. Holmes MD, Chen WY, Feskanich D, Kroenke CH, Colditz GA (2005) Physical activity and survival after breast cancer diagnosis. Jama 293(20):2479-2486

8. Holick CN, Newcomb PA, Trentham-Dietz A et al (2008) Physical activity and survival after diagnosis of invasive breast cancer. Cancer Epidemiol Biomarkers Prev 17(2):379-386 
9. Irwin ML, Smith AW, McTiernan A et al (2008) Influence of preand postdiagnosis physical activity on mortality in breast cancer survivors: the health, eating, activity, and lifestyle study. J Clin Oncol 26(24):3958-3964

10. Sternfeld B, Weltzien E, Quesenberry CP Jr et al (2009) Physical activity and risk of recurrence and mortality in breast cancer survivors: findings from the LACE study. Cancer Epidemiol Biomarkers Prev 18(1):87-95

11. Friedenreich CM, Gregory J, Kopciuk KA, Mackey JR, Courneya KS (2009) Prospective cohort study of lifetime physical activity and breast cancer survival. Int J Cancer 124(8):1954-1962

12. Pasanisi P, Berrino F, De Petris M, Venturelli E, Mastroianni A, Panico S (2006) Metabolic syndrome as a prognostic factor for breast cancer recurrences. Int J Cancer 119(1):236-238

13. Irwin ML, Varma K, Alvarez-Reeves M et al (2009) Randomized controlled trial of aerobic exercise on insulin and insulin-like growth factors in breast cancer survivors: the Yale Exercise and Survivorship study. Cancer Epidemiol Biomarkers Prev 18(1): 306-313

14. McTiernan A, Ulrich C, Slate S, Potter J (1998) Physical activity and cancer etiology: associations and mechanisms. Cancer Causes Control 9(5):487-509

15. Pierce JP, Faerber S, Wright FA et al (2002) A randomized trial of the effect of a plant-based dietary pattern on additional breast cancer events and survival: the Women's Healthy Eating and Living (WHEL) Study. Control Clin Trials 23(6):728-756

16. Pierce JP, Natarajan L, Caan BJ et al (2007) Influence of a diet very high in vegetables, fruit, and fiber and low in fat on prognosis following treatment for breast cancer: the Women's Healthy Eating and Living (WHEL) randomized trial. Jama 298(3): 289-298
17. Gold EB, Flatt SW, Pierce JP et al (2006) Dietary factors and vasomotor symptoms in breast cancer survivors: the WHEL Study. Menopause 13(3):423-433

18. Pierce JP, Stefanick ML, Flatt SW et al (2007) Greater survival after breast cancer in physically active women with high vegetable-fruit intake regardless of obesity. J Clin Oncol 25(17): 2345-2351

19. McTiernan A, Kooperberg C, White E et al (2003) Recreational physical activity and the risk of breast cancer in postmenopausal women: the Women's Health Initiative Cohort Study. Jama 290(10):1331-1336

20. Hong S, Bardwell WA, Natarajan L et al (2007) Correlates of physical activity level in breast cancer survivors participating in the Women's Healthy Eating and Living (WHEL) Study. Breast Cancer Res Treat 101(2):225-232

21. Ainsworth BE, Haskell WL, Whitt MC et al (2000) Compendium of physical activities: an update of activity codes and MET intensities. Med Sci Sports Exerc 32(9 Suppl):S498-S504

22. Johnson-Kozlow M, Rock CL, Gilpin EA, Hollenbach KA, Pierce JP (2007) Validation of the WHI brief physical activity questionnaire among women diagnosed with breast cancer. Am J Health Behav 31(2):193-202

23. Hays RD, Sherbourne CD, Mazel RM (1993) The RAND 36-item health survey 1.0. Health Econ 2(3):217-227

24. Nelson ME, Rejeski WJ, Blair SN et al (2007) Physical activity and public health in older adults: recommendation from the American College of Sports Medicine and the American Heart Association. Med Sci Sports Exerc 39(8):1435-1445 